\title{
OPTIMAL SHAPE REMODELLING OF LINEARLY ELASTIC PLATES USING FINITE ELEMENT METHODS
}

\author{
MOON-SOO NA, NOBORU KIKUCHI AND JOHN E. TAYLOR \\ The University of Michigan, Ann Arbor, Michigan, U.S.A.
}

\begin{abstract}
SUMMARY
The optimality conditions for the optimal shape remodelling of linearly elastic plates are obtained by introducing the total variation of a function defined on a variable domain, although the variation of a function has been taken on a fixed domain in most literature on calculus of variations. Using these optimality conditions, a solution scheme involving an iterative algorithm is proposed, together with several numerical examples.
\end{abstract}

\section{INTRODUCTION}

The problem of optimal shape design distinguishes the category of structural design problems where the objective is to predict optimal geometric configuration or layout for a structure. Prager's work ${ }^{1}$ includes developments on this type of problem among his broad contributions to various aspects of study in structural optimization. More material by Zienkiewicz and Campbell, ${ }^{2}$ Francavilla et al.,${ }^{3}$ Dems and Mroz, ${ }^{4}$ Haug et al.,${ }^{5}$ Ramakrishnan and Francavilla, ${ }^{6}$ Kristensen and Madsen, ${ }^{7}$ Chun and Haug ${ }^{8}{ }^{\text {Queau and Trompette }}{ }^{9}$ and Imam $^{10}$ reflects progress in the development of means for the formulation and computational solution of shape design problems. A thorough survey of the literature on shape optimization is included in the review article by Haug. ${ }^{11}$ Although a substantial amount of literature has been developed on the shape optimization problem, almost all of the studies are concerned with finding the optimal design within a constant volume or cost constraint, but without using the idea of remodelling of structures.

In this paper, a variational formulation is presented for the optimal shape design of twodimensional linearly elastic bodies. The idea of optimal structural remodelling ${ }^{12}$ is introduced to consider shape optimization, where the objective is to predict an optimal shape modification to a specified shape. Optimal remodelling refers to the selection of the best form of modification within an available amount of resource, and can be classified into three different formulations: (1) reinforcement remodelling in which the objective is to find optimal distribution of reinforcement (addition of material), (2) lightening remodelling in which the objective is to find the optimal distribution of removal of material, and (3) compound remodelling in which simultaneous removal and reinforcement of material (redistribution) may take place in an optimal way. This remodelling formulation seems to be broader and more practical in engineering application than conventional optimal structural design methods. Indeed, the conventional formulation can be regarded as a special case of this type of problem, i.e. of the compound remodelling problem, and it provides a way to modify existing structures. Moreover, the optimality conditions which will be derived below provide the basis for an approach to a 
computational method for such problems starting from the existing structures. Details of such a treatment of these problems can be found in the thesis of $\mathrm{Na}^{13}$ for plane stress problems as well as for torsion problems of a linearly elastic bar. Following $\mathrm{Na}^{13}$ we shall study shape optimization problems specifically for plane linear elasticity in this paper.

With the introduction of the remodelling functions, the optimality conditions are derived for compound remodelling along various types of boundary, according to the objective of minimizing mean compliance measured as the minimum total potential energy. Based on the derived optimality conditions, a computational scheme is proposed and applied to solve several example problems: it includes a case where the optimal modification calls for the introduction of a hole into an original shape specified as simply-connected. Finite element discretization is made in the stage of actual numerical computation.

\section{DESIGN PROBLEM FOR MINIMUM MEAN COMPLIANCE}

Consider a doubly-connected plane section of an elastic body, the thickness of which in the normal direction is assumed to be unity, as shown in Figure 1. In Figure 1, $\mathbf{u}$ is the displacement vector, $\hat{\mathbf{t}}$ the specified traction vector, $\hat{\mathbf{b}}$ the body-force vector, and $\mathbf{n}$ denotes a unit vector outward normal to the boundaries. An external boundary $\Gamma^{0}$ of a physical domain $\Omega$ is composed of the parts of the boundary $\Gamma_{t}^{0}$, where the traction vector $\hat{\mathbf{t}}$ is specified, $\Gamma_{u}^{0}$ where the displacement vector $\mathbf{u}$ is specified, and $\Gamma_{f}^{0}$ where the traction is zero, i.e. it is a traction-free boundary. Similarly, an internal boundary $\Gamma^{i}$ is composed of segments $\Gamma_{t}^{i}, \Gamma_{u}^{i}$ and $\Gamma_{f}^{i}$. The total potential energy of the body is given by

$$
\pi(\mathbf{u})=\frac{1}{2} \iint_{\Omega} \sigma_{i j}(\mathbf{u}) \varepsilon_{i j}(\mathbf{u}) \mathrm{d} \Omega-\iint_{\Omega} \hat{\mathbf{b}} \cdot \mathbf{u} \mathrm{d} \Omega-\int_{\left(\Gamma_{t}^{0}+\Gamma_{t}^{i}\right)} \hat{\mathbf{t}} \cdot \mathbf{u} \mathrm{d} \Gamma
$$

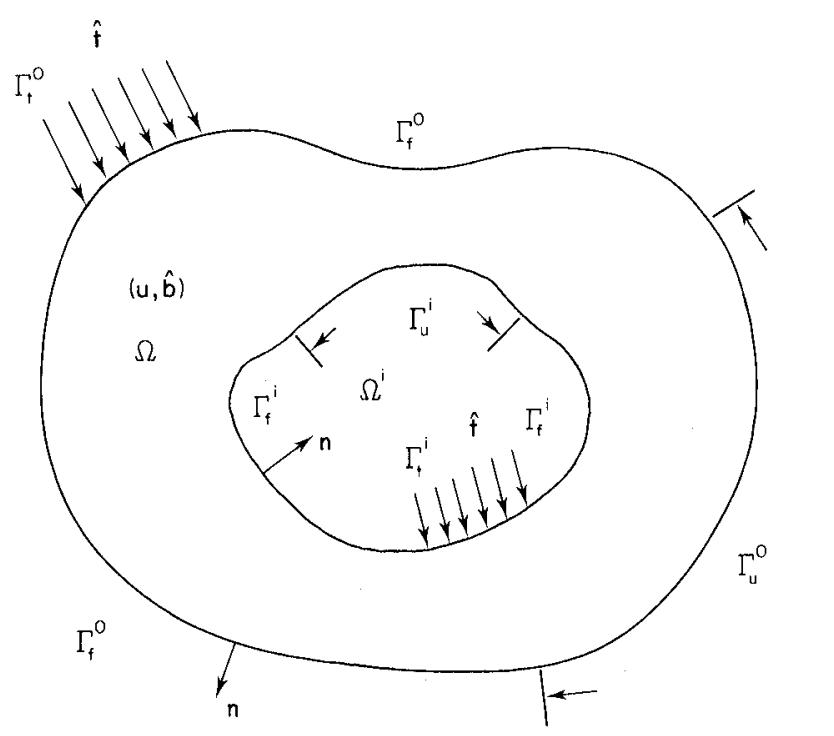

Figure 1. A doubly-connected plane section of an elastic body 
where $\boldsymbol{\sigma}=\left[\sigma_{i j}\right]$ is the stress tensor related to the strain tensor $\varepsilon=\left[\varepsilon_{i j}\right]$ by the constitutive equation

$$
\sigma_{i j}=E_{i j k l} \varepsilon_{k l}
$$

with

$$
E_{i j k l}=E_{k l i j}=E_{j i k l}=E_{i j l k}
$$

and strain $\varepsilon_{i j}$ given in terms of displacement $\mathbf{u}$ by

$$
\varepsilon_{i j}(\mathbf{u})=\left(u_{i, j}+u_{j, i}\right) / 2
$$

For prescribed displacement over portions $\Gamma_{u}^{0}$ and $\Gamma_{u}^{i}$ of the boundaries,

$$
\mathbf{u}=\hat{\mathbf{g}} \text { on } \Gamma_{u}^{0} \text { and } \Gamma_{u}^{i}
$$

The first variation of the total potential energy. with respect to the displacement field on the fixed domain $\Omega$ yields the virtual work principle:

$$
a_{\Omega}(\mathbf{u}, \mathbf{v})=f_{\Omega}(\mathbf{v}) \quad \forall \mathbf{v} \quad \text { s.t. } \mathbf{v}=\mathbf{0} \quad \text { on } \Gamma_{u}^{0} \text { and } \Gamma_{u}^{i}
$$

where $\mathbf{v}=\delta \mathbf{u}$ is the variation of $\mathbf{u}$ on the fixed domain $\Omega$, and

$$
\begin{aligned}
a_{\Omega}(\mathbf{u}, \mathbf{v}) & =\iint_{\Omega} E_{i j k l} \varepsilon_{k l}(\mathbf{u}) \varepsilon_{i j}(\mathbf{v}) \mathrm{d} \Omega \\
f_{\Omega}(\mathbf{v}) & =\iint_{\Omega} \hat{\mathbf{b}} \cdot \mathbf{v} \mathrm{d} \Omega+\int_{\left(\Gamma_{t}^{0}+\Gamma_{i}^{i}\right)} \hat{\mathbf{t}} \cdot \mathbf{v} \mathrm{d} \Gamma
\end{aligned}
$$

In (6) and (7), the subscript $\Omega$ is used to reflect the dependence of the functionals on the 'fixed' domain $\Omega$. Equation (6) further implies the equilibrium equation and the associated boundary conditions:

$$
\begin{aligned}
-\sigma_{i j}(\mathbf{u})_{, j} & =\hat{b}_{i} & & \text { in } \Omega \\
\sigma_{i j}(\mathbf{u}) n_{j} & =\hat{t}_{i} & & \text { on } \Gamma_{t}^{0} \text { and } \Gamma_{t}^{i} \\
\mathbf{u} & =\hat{\mathbf{g}} & & \text { on } \Gamma_{t}^{0} \text { and } \Gamma_{u}^{i}
\end{aligned}
$$

Now an optimal design problem is defined as follows: find the shape of a domain $\Omega$ that yields the minimum mean compliance

$$
\min _{\Omega \in M} f_{\Omega}(\mathbf{u})
$$

where $M$ is a proper admissible set of possible design domains. Since (6) yields

$$
\pi(\mathbf{u})=\min _{\mathbf{v} \in K_{\Omega}} \pi(\mathbf{v})=-\frac{1}{2} f_{\Omega}(\mathbf{u})
$$

the design problem is equivalently stated as

$$
\max _{\mathbf{\Omega} \in M} \min _{\mathbf{v} \in K_{\mathbf{\Omega}}} \pi(\mathbf{v})
$$

where $K_{\Omega}$ is the admissible set of displacement fields defined in the domain $\Omega$ which satisfy the kinematic boundary condition on $\Gamma_{u}^{0}$ and $\Gamma_{u}^{i}$.

The admissible set $M$ of possible design domains is in general restricted by an isoparametric relation in their measure, and will be defined more precisely in the following section. 


\section{REMODELLING FUNCTIONS TO DEFINE THE DESIGN DOMAIN}

In order to identify a doubly-connected domain in the polar co-ordinate system, the radius functions $R_{0}^{0}(\theta)$ and $R_{0}^{i}(\theta)$ are introduced to define the external boundary $\Gamma_{0}^{0}$ and the internal boundary $\Gamma_{0}^{i}$, respectively, of a given doubly-connected domain $\Omega_{0}$. The external and internal boundaries $\Gamma^{0}$ and $\Gamma^{i}$ of a modified domain $\Omega$ are identified in turn via the radius functions $R^{0}(\theta)$ and $R^{i}(\theta)$. It is assumed that all the possible design domains have star-shaped external and internal boundaries, as shown in Figure 2; that is, for $\Gamma_{0}^{0}$ (and $\Gamma^{0}$ ) there exists a point $\left(X_{c r}^{0}, Y_{c r}^{0}\right)$ such that any radial segment intersects the boundary $\Gamma_{0}^{0}\left(\right.$ and $\left.\Gamma^{0}\right)$ just once. Similarly, for $\Gamma_{0}^{i}\left(\right.$ and $\left.\Gamma^{i}\right)$ such a point $\left(X_{c r}^{i}, Y_{c r}^{i}\right)$ can also be found.

The remodelling functions are then defined in terms of the radius functions according to:

$$
\begin{array}{ll}
R^{0}(\theta)=R_{0}^{0}(\theta)+\rho_{r}^{0}(\theta)-\rho_{l}^{0}(\theta) & \forall \theta \\
R^{i}(\theta)=R_{0}^{i}(\theta)-\rho_{r}^{i}(\theta)+\rho_{l}^{i}(\theta) & \forall \theta
\end{array}
$$

The functions $\rho_{r}^{0}(\theta)$ and $\rho_{l}^{0}(\theta)$, which are orthogonal to each other, define reinforcement remodelling and lightening remodelling, respectively, along the external boundary. The similar definitions are made to the functions $\rho_{r}^{i}(\theta)$ and $\rho_{l}^{i}(\theta)$ along the internal boundary.

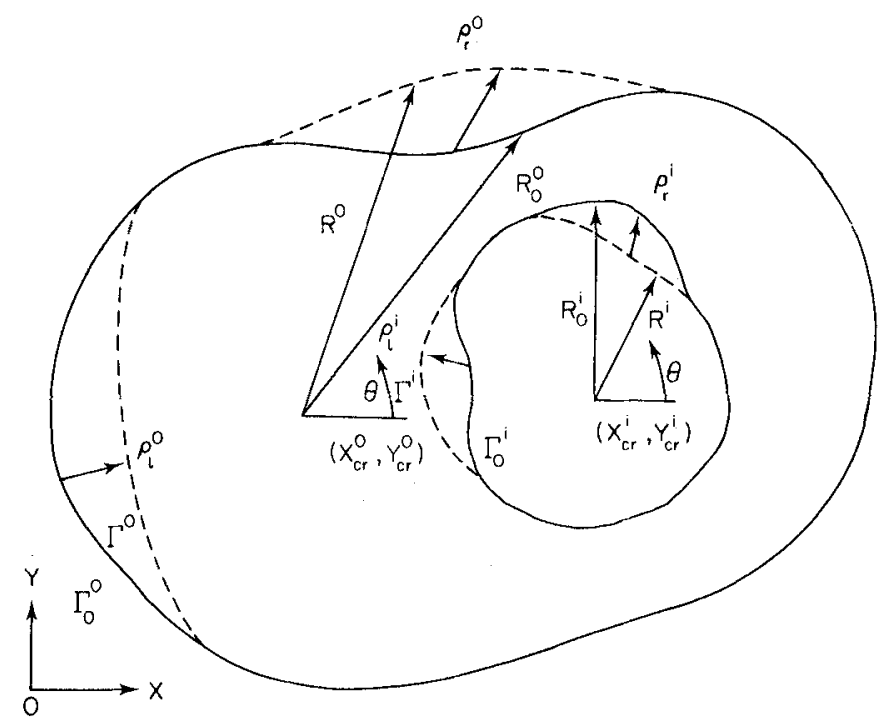

Figure 2. Domain identification through remodelling functions

Note that if $\rho_{r}^{0}(\theta) \geqslant 0$ and $\rho_{l}^{0}(\theta) \geqslant 0 \forall \theta$ along the external boundary, we have $R^{0}(\theta) \geqslant R_{0}^{0}(\theta)$ for intervals of reinforcement, while $R^{0}(\theta) \leqslant R_{0}^{0}(\theta)$ wherever the domain is reduced. Similarly, by constraining as $\rho_{r}^{i}(\theta) \geqslant 0$ and $\rho_{l}^{i}(\theta) \geqslant 0 \forall \theta$ along the internal boundary, the conditions $R^{i}(\theta) \leqslant R_{0}^{i}(\theta)$ or $R^{i}(\theta) \geqslant R_{0}^{i}(\theta)$ are satisfied for reinforcement or for lightening, respectively.

The net amount of material volume for reinforcement, say $V_{+}$, and for lightening, say $V_{-}$, can be represented by

$$
\begin{aligned}
& V_{+}\left(\rho_{r}^{0}, \rho_{r}^{i}\right)=\frac{1}{2} \int_{0}^{2 \pi} \rho_{r}^{0}\left(2 R_{0}^{0}+\rho_{r}^{0}\right) \mathrm{d} \theta+\frac{1}{2} \int_{0}^{2 \pi} \rho_{r}^{i}\left(2 R_{0}^{i}-\rho_{r}^{i}\right) \mathrm{d} \theta \\
& V_{-}\left(\rho_{l}^{0}, \rho_{l}^{i}\right)=\frac{1}{2} \int_{0}^{2 \pi} \rho_{l}^{0}\left(2 R_{0}^{0}-\rho_{l}^{0}\right) \mathrm{d} \theta+\frac{1}{2} \int_{0}^{2 \pi} \rho_{l}^{i}\left(2 R_{0}^{i}+\rho_{l}^{i}\right) \mathrm{d} \theta
\end{aligned}
$$


If compound remodelling is considered, the design modifications are constrained via

$$
V_{-}\left(\rho_{l}^{0}, \rho_{l}^{i}\right) \geqslant \gamma V_{0}, \quad V_{+}\left(\rho_{r}^{0}, \rho_{r}^{i}\right) \leqslant \gamma V_{0}
$$

for given number $0<\gamma<1$, where $V_{0}$ is the material volume of the original elastic body. Note that there is no explicit restriction on where material is to be added or removed along the boundaries.

If only a reinforcement remodelling is to be considered, the maximum amount of material to be added is restricted as in (14), i.e.

Similarly, for lightening only

$$
V_{+}\left(\rho_{r}^{0}, \rho_{r}^{i}\right) \leqslant \gamma V_{0}
$$

$$
V_{-}\left(\rho_{l}^{0}, \rho_{l}^{i}\right) \geqslant \gamma V_{0}
$$

\section{OPTIMALITY CONDITIONS}

A compound remodelling only along the external boundary is considered as an example problem to explain the basis for both reinforcement and lightening remodellings. Once the optimality conditions are obtained for a compound remodelling along the external boundary, the method to derive the optimality conditions can easily be extended to any remodelling problems along the internal boundary. Details for such more general remodelling problems can be found in $\mathrm{Na}^{13}$ The whole external boundary is subjected to be designed for obtaining optimality conditions, although a special case that the design is limited only along the traction-free boundary will be considered as a concrete example of a shape optimization problem.

The optimal design problem (11) is now defined in terms of the remodelling function $\boldsymbol{\rho}=\left\{\rho_{r}^{0}, \rho_{l}^{0}\right\}$ as

$$
\max _{\mathbf{p} \in M} \min _{\mathbf{v} \in K_{\mathbf{p}}} \pi(\mathbf{v})
$$

where $K_{\mathbf{p}}=K_{\Omega(\boldsymbol{p})}$ and the set $M$ is designed such that $\mathbf{p} \in M$ if

$$
\begin{array}{rc}
-\rho_{r}^{0}(\theta) \leqslant 0 & \forall \theta \\
-\rho_{l}^{0}(\theta) \leqslant 0 & \forall \theta \\
\rho_{r}^{0}(\theta) \rho_{l}^{0}(\theta)=0 & \forall \theta \\
-V_{-}\left(\rho_{l}^{0}, 0\right)+\gamma V_{0} \leqslant 0 & \\
V_{+}\left(\rho_{r}^{0}, 0\right)-\gamma V_{0} \leqslant 0 &
\end{array}
$$

Here, $V_{0}$ and $\gamma$ are given a priori, and the notation $\Omega(\boldsymbol{\rho})$ is used to show dependence of the domain $\Omega$ on the remodelling function $\rho$. Introducing the Lagrange multipliers $\boldsymbol{\lambda}=$ $\left\{\lambda_{r}, \lambda_{l}, \lambda_{0}, \lambda_{s}, \lambda_{a}\right\}$ to the constraints on the remodelling functions, the Lagrangian is defined as

$$
\begin{aligned}
L(\boldsymbol{\rho}, \boldsymbol{\lambda})= & \pi(\boldsymbol{\rho}, \mathbf{u})+\int_{0}^{2 \pi} \lambda_{r} \rho_{r}^{0} \mathrm{~d} \theta+\int_{0}^{2 \pi} \lambda_{l} \rho_{l}^{0} \mathrm{~d} \theta \\
& -\int_{0}^{2 \pi} \lambda_{0} \rho_{r}^{0} \rho_{l}^{0} \mathrm{~d} \theta-\lambda_{s}\left(-V_{-}+\gamma V_{0}\right)-\lambda_{a}\left(V_{+}-\gamma V_{0}\right)
\end{aligned}
$$

where $\mathbf{u}$ is the solution to the minimization problem

$$
\min _{\mathbf{v} \in K_{\mathbf{p}}} \pi(\mathbf{v})
$$

and $\lambda_{r} \geqslant 0, \lambda_{l} \geqslant 0, \lambda_{s} \geqslant 0$ and $\lambda_{a} \geqslant 0$. 
For the variation of the total potential energy $\pi(\boldsymbol{\rho}, \mathbf{u})$ with respect to $\boldsymbol{\rho}$, it is necessary to consider the variation of the displacement $\mathbf{u}$ due to change of the domain, especially along the boundary. Let $\delta \mathbf{u}$ denote the variation of the displacement $\mathbf{u}$ for the domain defined by the remodelling function $\boldsymbol{\rho}$, and let $\delta_{t} \mathbf{u}$ represent the total variation of $\mathbf{u}$ due to the variation of p. The relationships between $\delta_{t} \mathbf{u}$ and $\delta \mathbf{u}$ are given as follows:

$$
\delta_{t} u_{i}=\delta u_{i}+u_{i, j} \delta_{j} p \quad \text { on } \Gamma^{0}
$$

where

$$
\begin{aligned}
& \delta_{1} p=\left(\delta \rho_{r}^{0}-\delta \rho_{l}^{0}\right) \cos \theta \\
& \delta_{2} p=\left(\delta \rho_{r}^{0}-\delta \rho_{l}^{0}\right) \sin \theta
\end{aligned}
$$

The boundary conditions in (8) require that

$$
\delta_{t} \mathbf{u}=\mathbf{0} \quad \text { on } \Gamma_{u}^{0}
$$

and $\delta_{t} \mathbf{u}$ is arbitrary on both $\Gamma_{t}^{0}$ and $\Gamma_{f}^{0}$. Thus, the relation (21) leads to

$$
\begin{array}{ll}
\delta u_{i}=\delta_{t} u_{i}-u_{i, j} \delta_{j} p & \text { on } \Gamma_{t}^{0} \text { and } \Gamma_{f}^{0} \\
\delta u_{i}=-u_{i, j} \delta_{j} p & \text { on } \Gamma_{u}^{0}
\end{array}
$$

We suppose here that the traction along the boundary $\Gamma_{\mathfrak{t}}^{0}$ is independent of the shape change of the domain.

Taking the variation of the Lagrangian (19) with respect to the remodelling function $\rho$ gives

$$
\begin{aligned}
\delta_{\boldsymbol{\rho}} L(\boldsymbol{\rho}, \boldsymbol{\lambda})= & \delta_{\boldsymbol{\rho}} \pi(\boldsymbol{\rho}, \mathbf{u})+\int_{0}^{2 \pi} \lambda_{r} \delta \rho_{r}^{0} \mathrm{~d} \theta+\int_{0}^{2 \pi} \lambda_{l} \delta \rho_{l}^{0} \mathrm{~d} \theta \\
& -\int_{0}^{2 \pi} \lambda_{0}\left(\rho_{l}^{0} \delta \rho_{r}^{0}+\rho_{r}^{0} \delta \rho_{l}^{0}\right) \mathrm{d} \theta \\
& -\lambda_{a} \int_{0}^{2 \pi}\left(R_{0}^{0}+\rho_{r}^{0}\right) \delta \rho_{r}^{0} \mathrm{~d} \theta+\lambda_{s} \int_{0}^{2 \pi}\left(R_{0}^{0}-\rho_{l}^{0}\right) \delta \rho_{l}^{0} \mathrm{~d} \theta
\end{aligned}
$$

where

$$
\begin{aligned}
\delta_{\mathbf{p}} \pi(\boldsymbol{\rho}, \mathbf{u})= & -\iint_{\Omega}\left[\sigma_{i j}(\mathbf{u})_{, j}+\hat{b}_{i}\right] \delta u_{i} \mathrm{~d} \Omega \\
& +\int_{\Gamma^{0}} \sigma_{i j}(\mathbf{u}) n_{j} \delta u_{i} \mathrm{~d} \Gamma+\int_{\Gamma^{i}} \sigma_{i j}(\mathbf{u}) n_{j} \delta u_{i} \mathrm{~d} \Gamma \\
& -\int_{\Gamma_{t}^{0}} \hat{t_{i}} \delta u_{i} \mathrm{~d} \Gamma-\int_{\Gamma_{t}^{i}} \hat{t}_{i} \delta u_{i} \mathrm{~d} \Gamma \\
& +\int_{\Gamma^{0}}\left[\frac{1}{2} \sigma_{i j}(\mathbf{u}) \varepsilon_{i j}(\mathbf{u})-\hat{b}_{i} u_{i}\right] \delta_{n} p \mathrm{~d} \Gamma \\
& -\int_{\Gamma_{t}^{0}(\theta)} \hat{t}_{i} u_{i}\left(\delta \rho_{r}^{0}-\delta \rho_{l}^{0}\right) \mathrm{d} \theta
\end{aligned}
$$

Here, $\delta_{n} p=\delta_{j} p n_{j}, \Gamma^{0}=\Gamma_{t}^{0} \cup \Gamma_{f}^{0} \cup \Gamma_{u}^{0}, \Gamma^{i}=\Gamma_{t}^{i} \cup \Gamma_{f}^{i} \cup \Gamma_{u}^{i}$ and $\Gamma_{t}^{0}(\theta)$ denotes the intervals (or the unions of intervals) in the polar co-ordinate system identifying the part of the boundary $\Gamma_{t}^{0}$. 
Note that the following equivalence expression has been used:

$$
\int_{\Gamma_{\mathrm{r}}^{0}} \hat{t}_{i} u_{i} \mathrm{~d} \Gamma=\int_{\Gamma_{i}^{0}(\theta)} \hat{t}_{i} u_{i}\left(R_{0}^{0}+\rho_{r}^{0}-\rho_{l}^{0}\right) \mathrm{d} \theta
$$

Using the relations (8) and (22), and enforcing the relations on the variation of remodelling functions such that

$$
\delta_{n} p \mathrm{~d} \Gamma=\left(R_{0}^{0}+\rho_{r}^{0}-\rho_{l}^{0}\right)\left(\delta \rho_{r}^{0}-\delta \rho_{l}^{0}\right) \mathrm{d} \theta \quad \text { on } \Gamma^{0}
$$

the expression for $\delta_{\mathbf{p}} \pi(\boldsymbol{\rho}, \mathbf{u})$ is simplified to

$$
\begin{aligned}
\delta_{\rho} \pi(\boldsymbol{\rho}, \mathbf{u})= & \int_{\Gamma_{\mathrm{f}}^{0}(\theta)}\left[U(\mathbf{u})-\hat{b}_{i} u_{i}\right]\left(R_{0}^{0}+\rho_{r}^{0}-\rho_{l}^{0}\right)\left(\delta \rho_{r}^{0}-\delta \rho_{l}^{0}\right) \mathrm{d} \theta \\
& +\int_{\Gamma_{u}^{0}(\theta)}\left[U(\mathbf{u})-\sigma_{i j}(\mathbf{u}) n_{j}\left(u_{i, 1} \cos \theta+u_{i, 2} \sin \theta\right)\right. \\
& \left.-\hat{b}_{i} u_{i}\right]\left(R_{0}^{0}+\rho_{r}^{0}-\rho_{l}^{0}\right)\left(\delta \rho_{r}^{0}-\delta \rho_{l}^{0}\right) \mathrm{d} \theta \\
& +\int_{\Gamma_{t}^{0}(\theta)}\left\{\left[U(\mathbf{u})-\hat{b}_{i} u_{i}\right]\left(R_{0}^{0}+\rho_{r}^{0}-\rho_{l}^{0}\right)-\hat{t}_{i} u_{i}\right\}\left(\delta \rho_{r}^{0}-\delta \rho_{l}^{0}\right) \mathrm{d} \theta
\end{aligned}
$$

Here

$$
U(\mathbf{u})=\frac{1}{2} \sigma_{i j}(\mathbf{u}) \varepsilon_{i j}(\mathbf{u})
$$

denotes the specific strain energy, i.e. strain energy per unit volume. Thus, from (23) and (26), $\delta_{\boldsymbol{\rho}} L(\boldsymbol{\rho}, \boldsymbol{\lambda})=0$ yields the following conditions to be satisfied by the optimal remodelling:

1. For reinforcement remodelling $\rho_{r}^{0}$

$$
\begin{gathered}
{\left[U(\mathbf{u})-\hat{b}_{i} u_{i}\right]\left(R_{0}^{0}+\rho_{r}^{0}-\rho_{l}^{0}\right)-\lambda_{a}\left(R_{0}^{0}+\rho_{r}^{0}\right)+\lambda_{r}-\lambda_{0} \rho_{l}^{0}=0 \quad \forall \theta \in \Gamma_{f}^{0}(\theta)} \\
{\left[U(\mathbf{u})-\sigma_{i j}(\mathbf{u}) n_{j}\left(u_{i, 1} \cos \theta+u_{i, 2} \sin \theta\right)-\hat{b}_{i} u_{i}\right]\left(R_{0}^{0}+\rho_{r}^{0}-\rho_{l}^{0}\right)-\lambda_{a}\left(R_{0}^{0}+\rho_{r}^{0}\right)+\lambda_{r}-\lambda_{0} \rho_{l}^{0}=0} \\
\forall \theta \in \Gamma_{u}^{0}(\theta) \\
{\left[U(\mathbf{u})-\hat{b}_{i} u_{i}\right]\left(R_{0}^{0}+\rho_{r}^{0}-\rho_{l}^{0}\right)-\hat{t}_{i} u_{i}-\lambda_{a}\left(R_{0}^{0}+\rho_{r}^{0}\right)+\lambda_{r}-\lambda_{0} \rho_{l}^{0}=0 \quad \forall \theta \in \Gamma_{t}^{0}(\theta)}
\end{gathered}
$$

2. For lightening remodelling $\rho_{l}^{0}$

$$
\begin{gathered}
-\left[U(\mathbf{u})-\hat{b}_{i} u_{i}\right]\left(R_{0}^{0}+\rho_{r}^{0}-\rho_{l}^{0}\right)+\lambda_{s}\left(R_{0}^{0}-\rho_{l}^{0}\right)+\lambda_{l}-\lambda_{0} \rho_{r}^{0}=0 \quad \forall \theta \in \Gamma_{f}^{0}(\theta) \\
{\left[U(\mathbf{u})-\sigma_{i j}(\mathbf{u}) n_{j}\left(u_{i, 1} \cos \theta+u_{i, 2} \sin \theta\right)-\hat{b}_{i} u_{i}\right]\left(R_{0}^{0}+\rho_{r}^{0}-\rho_{l}^{0}\right)+\lambda_{s}\left(R_{0}^{0}-\rho_{l}^{0}\right)+\lambda_{l}-\lambda_{0} \rho_{r}^{0}=0} \\
\forall \theta \in \Gamma_{u}^{0}(\theta) \\
-\left[U(\mathbf{u})-\hat{b}_{i} u_{i}\right]\left(R_{0}^{0}+\rho_{r}^{0}-\rho_{l}^{0}\right)+\hat{t}_{i} u_{i}+\lambda_{s}\left(R_{0}^{0}-\rho_{l}^{0}\right)+\lambda_{l}-\lambda_{0} \rho_{r}^{0}=0 \quad \forall \theta \in \Gamma_{t}^{0}(\theta)
\end{gathered}
$$

Taking the variations of $L(\rho, \lambda)$ with respect to the Lagrange multipliers produces the conditions

$$
\begin{aligned}
\lambda_{r} \geqslant 0, \quad \lambda_{r} \rho_{r}^{0}=0 & & \text { on } \Gamma^{0} \\
\lambda_{l} \geqslant 0, \quad \lambda_{l} \rho_{l}^{0}=0 & & \text { on } \Gamma^{0} \\
\lambda_{0} \rho_{r}^{0} \rho_{l}^{0}=0 & & \text { on } \Gamma^{0} \\
\lambda_{s} \geqslant 0, \quad \lambda_{s}\left(-V_{-}+\gamma V_{0}\right) & =0 & \\
\lambda_{a} \geqslant 0, \quad \lambda_{a}\left(V_{+}-\gamma V_{0}\right) & =0 &
\end{aligned}
$$


In order to obtain the meaning of the derived optimality conditions, let us consider the special case of compound remodelling along the free boundary when body forces are absent, i.e. $\hat{\mathbf{b}}=\mathbf{0}$. Define

$$
\begin{aligned}
& I_{r}=\left\{\theta: \theta \in \Gamma_{f}^{0}(\theta) \text { and } \rho_{r}^{0}(\theta)>0\right\} \\
& I_{l}=\left\{\theta: \theta \in \Gamma_{f}^{0}(\theta) \text { and } \rho_{l}^{0}(\theta)>0\right\}
\end{aligned}
$$

From the relations (28) and (34)-(36),

$$
\begin{aligned}
U(\mathbf{u}) & =\lambda_{a} \quad \forall \theta \in I_{r} \\
\lambda_{r} & =\left[\lambda_{a}-U(\mathbf{u})\right] R_{0}^{0} \quad \forall \theta \notin I_{r} \text { and } \theta \notin I_{l}
\end{aligned}
$$

From the relations (31) and (34)-(36),

$$
\begin{aligned}
U(\mathbf{u}) & =\lambda_{s} \quad \forall \theta \in I_{l} \\
\lambda_{l} & =\left[U(\mathbf{u})-\lambda_{s}\right] R_{0}^{0} \quad \forall \theta \notin I_{r} \text { and } \theta \notin I_{l}
\end{aligned}
$$

It can be said from (40) and (41) that the specific strain energy distribution $U(\mathbf{u})$ is constant along the parts of the free external boundary where design modifications are made in an optimal way, and that the constant $\lambda_{a}$ provides an upper bound and the constant $\lambda_{s}$ provides a lower bound to the value of $U(\mathbf{u})$ along the free external boundary. This further implies that

$$
\lambda_{a}>0 \text { and } \lambda_{s}>0
$$

so that the relations (37) and (38) lead to

$$
V_{-}=\gamma V_{0} \text { and } \quad V_{+}=\gamma V_{0}
$$

at the optimally modified domain.

The same procedure is applicable to other remodelling problems, even for a multiplyconnected domain.

\section{A SOLUTION SCHEME}

For simplicity of discussion, the compound remodelling along the free boundary is considered when body forces are absent. The solution procedure to be described here is based upon the meaning of the derived optimality conditions (28)-(38), i.e. (39)-(43) for the present case. Although it is possible to use the optimality conditions (28)-(38) for a solution scheme, for example, for the so-called Uzawa method, ${ }^{14}$ to find saddle points of a given Lagrangian, the use of the conditions (39)-(43) is, in general, expected to give rather fast convergence to the solution. The main idea of the solution method is to obtain the values of two positive constants $\lambda_{a}$ and $\lambda_{s}$ which should be determined such that the relation (43) is satisfied and which also bound the distribution $U(\mathbf{u})$ both from above and from below. At the same time, the distribution $U(\mathbf{u})$ should be constant along the parts of the free boundary where design is modified.

An iterative solution procedure is given as follows. Let $\rho_{r}^{0(m)}, \rho_{l}^{o(m)}, \lambda_{a}^{(m)}, \lambda_{s}^{(m)}, I_{r}^{(m)}$ and $I_{l}^{(m)}$ identify the $m$ th iterates of the respective functions and quantities. Suppose that these iterates are determined so that the relations (34)-(36), (39) and (43) are satisfied. Define

$$
\begin{aligned}
& \rho_{r}^{0(m+1)}=P_{+}\left(\rho_{r}^{0(m)}+d_{r}^{0(m)}\right) \\
& \rho_{l}^{0(m+1)}=P_{+}\left(\rho_{l}^{0(m)}+d_{l}^{0(m)}\right)
\end{aligned}
$$

where $P_{+}$denotes a projection by which the $(m+1)$ th iterates of the remodelling functions $\rho_{r}^{0(m+1)}$ and $\rho_{l}^{0(m+1)}$ are formed to satisfy the condition of being non-negative. Here, $d_{r}^{0(m)}$ and 
$d_{l}^{(m)}$ represent the design correction functions to be defined. Consider change $\Delta U$ with respect to a domain change represented by $\Delta R$. There would be a positive real number $\hat{\delta}$ such that in the polar co-ordinate system the change $\Delta U$ is expressed as

$$
\Delta U(\theta)=\hat{\delta}\left|\frac{\partial U(r, \theta)}{\partial r}\right|_{\Gamma_{f}^{0}} \mid \Delta R(\theta) \quad \forall \theta \in \Gamma_{f}^{0}(\theta)
$$

To avoid the evaluation of the first-order partial derivative of $U(\mathbf{u})$ along the boundary, a modification of the relation (45) is used:

$$
\Delta U(\theta)=\beta \frac{U(\theta)}{R(\theta)} \Delta R(\theta) \quad \forall \theta \in \Gamma_{f}^{0}(\theta)
$$

where $\beta$ is a positive real number. Inversion of the relation (46) produces an expression for the domain change along the radial direction $\Delta R$ required to obtain a necessary amount of change $\Delta U$ at a boundary

$$
\Delta R(\theta)=\delta \frac{\Delta U(\theta)}{U(\theta)} R(\theta) \quad \forall \theta \in \Gamma_{f}^{0}(\theta)
$$

where $\delta$ is a positive real number. Since the distribution $U(\theta)$ should be constant along the parts of the free boundary where design is modified in an optimal way, identifying $\Delta U$ with the difference between $U$ and the constant value which is assumed temporarily as known leads to the definition of $\Delta R$ with required design correction. Thus, the design corrections are defined by

$$
\begin{aligned}
& d_{r}^{0(m)}=\delta_{r}^{0(m)} R_{(m)}^{0}\left(U^{(m)}-\lambda_{a}^{(m+1)}\right) / U^{(m)} \\
& d_{l}^{0(m)}=\delta_{l}^{0(m)} R_{(m)}^{0}\left(\lambda_{s}^{(m+1)}-U^{(m)}\right) / U^{(m)}
\end{aligned}
$$

where $\delta_{r}^{O(m)}$ and $\delta_{l}^{0(m)}$ are positive real numbers, $R_{(m)}^{0}$ is the $m$ th iterate of the radius function $R^{0}$, and $U^{(m)}$ is the $m$ th iterate of a specific strain energy distribution along the free external boundary. The $(m+1)$ th iterate values for two constants $\lambda_{a}^{(m+1)}$ and $\lambda_{s}^{(m+1)}$ are expected to be an upper bound and a lower bound, respectively, to the $(m+1)$ th iterate of the distribution $U$. These values are to be determined as the $(m+1)$ th iterates of the remodelling functions $\rho_{r}^{0(m+1)}$ and $\rho_{l}^{O(m+1)}$ defined in the relation (44) satisfy the relation (43).

For each iteration step, the computation of two constants $\lambda_{a}^{(m+1)}$ and $\lambda_{s}^{(m+1)}$ requires an inner loop of iteration, since the sets $I_{r}^{(m+1)}$ and $I_{l}^{(m+1)}$ are unknown. Define

$$
\begin{aligned}
& J_{r}^{(m)}=\left\{\theta: \theta \in \Gamma_{f}^{0}(\theta), \theta \in I_{r}^{(m)} \text { and } \theta \notin I_{r}^{(m+1)}\right\} \\
& J_{l}^{(m)}=\left\{\theta: \theta \in \Gamma_{f}^{0}(\theta), \theta \in I_{l}^{(m)} \text { and } \theta \notin I_{l}^{(m+1)}\right\}
\end{aligned}
$$

and

$$
\begin{aligned}
& \hat{V}_{+}^{(m)}=\frac{1}{2} \int_{J_{r}^{(m)}} \rho_{r}^{0(m)}\left(2 R_{0}^{0}+\rho_{r}^{0(m)}\right) \mathrm{d} \theta \\
& \hat{V}_{-}^{(m)}=\frac{1}{2} \int_{Y_{l}^{(m)}} \rho_{l}^{0(m)}\left(2 R_{0}^{0}-\rho_{l}^{0(m)}\right) \mathrm{d} \theta
\end{aligned}
$$


Substitution of the relation (44) into the relation (43) with use of the relations (49) and (50) leads to

$$
\begin{aligned}
& \frac{1}{2} \int_{I_{r}^{(m+1)}} d_{l}^{0(m)}\left[2\left(R_{0}^{0}+\rho_{r}^{0(m)}\right)+d_{r}^{0(m)}\right] \mathrm{d} \theta=\hat{V}_{+}^{(m)} \\
& \frac{1}{2} \int_{I_{l}^{(m+1)}} d_{l}^{O(m)}\left[2\left(R_{0}^{0}-\rho_{l}^{0(m)}\right)-d_{l}^{0(m)}\right] \mathrm{d} \theta=\hat{V}_{-}^{(m)}
\end{aligned}
$$

Substitution of the relation (48) into (51) yields two quadratic algebraic equations for two constants $\lambda_{a}^{(m+1)}$ and $\lambda_{s}^{(m+1)}$. It is necessary to choose one solution for each constant, which is within the range of consideration of a current distribution $U^{(m)}$.

The solution procedure can be summarized as follows:

Initialization: $m=0$

1.

$$
\rho_{r}^{0(0)}=\rho_{l}^{0(0)}=0 \quad \forall \theta \in \Gamma_{f}^{0}(\theta)
$$

and appropriate values are taken for $\lambda_{a}^{(0)}$ and $\lambda_{s}^{(0)}$.

Iteration: $m \leftarrow m+1$

2. Choose proper magnification factors $\delta_{r}^{0(m)}$ and $\delta_{l}^{0(m)}$.

3. Solve the boundary-value problem (8) by discretizing the weak form (7) for the domain identified with the remodelling functions $\rho_{r}^{0(m)}$ and $\rho_{l}^{0(m)}$, and compute the specific strain energy distribution

$$
U^{(m)}=\frac{1}{2} \sigma_{i j}\left(\mathbf{u}^{(m)}\right) \varepsilon_{i j}\left(\mathbf{u}^{(m)}\right)
$$

along the free external boundary.

4. Predict the constant specific strain energy levels $\lambda_{a}^{(m+1)}$ and $\lambda_{s}^{(m+1)}$ by the following inner iteration scheme:

4.1. Set $\lambda_{a(0)}^{(m+1)}=\lambda_{a}^{(m)}$ and $\lambda_{s(0)}^{(m+1)}=\lambda_{s}^{(m)}$, where the subscript in parentheses means the inner iteration step.

Inner iteration: $k \leftarrow k+1$

4.2. Identify $I_{r(k)}^{(m+1)}$ and $I_{l(k)}^{(m+1)}$ with $\lambda_{a(k-1)}^{(m+1)}$ and $\lambda_{s(k-1)}^{(m+1)}$ from $U^{(m)}$ such that

$$
I_{r(k)}^{(m+1)} \supseteq I_{r}^{(m)} \quad \text { and } \quad I_{l(k)}^{(m+1)} \supseteq I_{l}^{(m)}
$$

4.3. Compute $\lambda_{a(k)}^{(m+1)}$ and $\lambda_{s(k)}^{(m+1)}$ from the relation (51) by substitution of the relation (48) where $\lambda_{a}^{(m+1)}$ and $\lambda_{s}^{(m+1)}$ are replaced by $\lambda_{a(k)}^{(m+1)}$ and $\lambda_{s(k)}^{(m+1)}$, respectively.

4.4. Compare $\lambda_{a(k)}^{(m+1)}$ with $\lambda_{a(k-1)}^{(m+1)}$, and $\lambda_{s(k)}^{(m+1)}$ with $\lambda_{s(k-1)}^{(m+1)}$. If the differences in their iterate values are less than a specified amount, set $\lambda_{a}^{(m+1)}=\lambda_{a(k)}^{(m+1)}$ and $\lambda_{s}^{(m+1)}=\lambda_{s(k)}^{(m+1)}$. Otherwise go back to step 4.2 .

5. Compute $d_{r}^{0(m)}$ and $d_{i}^{0(m)}$ from (48), and obtain the new remodelling functions $\rho_{r}^{0(m+1)}$ and $\rho_{l}^{0(m+1)}$. Identify the sets $I_{r}^{(m+1)}$ and $I_{l}^{(m+1)}$ corresponding to $\rho_{r}^{0(m+1)}$ and $\rho_{l}^{0(m+1)}$.

6. Check the difference $\left(\rho_{r}^{0(m+1)}-\rho_{r}^{0(m)}\right),\left(\rho_{l}^{0(m+1)}-\rho_{l}^{0(m)}\right),\left(\lambda_{a}^{(m+1)}-\lambda_{a}^{(m)}\right)$ and $\left(\lambda_{s}^{(m+1)}-\lambda_{s}^{(m)}\right)$, and the change in the value of the objective function. If these are small enough and constancy of $U^{(m)}$ on the intervals $I_{r}^{(m)}$ and $I_{l}^{(m)}$ is achieved to satisfy the optimality conditions, then the process is completed. Otherwise, go back to step 2 . 
While Uzawa's method assumes the iteration for $\rho_{r}^{0}, \rho_{l}^{0}, \lambda_{a}$ and $\lambda_{s}$ using the specific strain energy

$$
\begin{aligned}
\rho_{r}^{0(m+1)} & =\max \left\{0, \rho_{r}^{0(m)}+C_{r}^{(m)}\left(U^{(m)}-\lambda_{a}^{(m)}\right)\right\} \\
\rho_{l}^{0(m+1)} & =\max \left\{0, \rho_{l}^{0(m)}-C_{l}^{(m)}\left(U^{(m)}-\lambda_{s}^{(m)}\right)\right\} \\
\lambda_{a}^{(m+1)} & =\max \left\{0, \lambda_{a}^{(m)}+C_{a}^{(m)}\left(V_{+}^{(m)}-\gamma V_{0}\right)\right\} \\
\lambda_{l}^{(m+1)} & =\max \left\{0, \lambda_{s}^{(m)}-C_{s}^{(m)}\left(V_{-}^{(m)}-\gamma V_{0}\right)\right\}
\end{aligned}
$$

for properly chosen constants $C_{r}^{(m)}, C_{l}^{(m)}, C_{a}^{(m)}$ and $C_{s}^{(m)}$, the solution procedure described in this work has the inner iteration loop to find the energy levels $\lambda_{a}$ and $\lambda_{s}$ such that the global constraints, $V_{+}=\gamma V_{0}$ and $V_{-}=\gamma V_{0}$, are exactly satisfied in each iteration step. In general, sufficiently small constants $C_{a}^{(m)}$ and $C_{s}^{(m)}$ have to be taken in Uzawa's method (53), and this leads to rather slow convergence since the design correction on $\rho_{r}^{0}$ and $\rho_{l}^{0}$ strongly depend up the energy levels $\lambda_{a}$ and $\lambda_{s}$. However, the present scheme proposed resolves this disadvantage of Uzawa's method by using the inner loop to find the energy levels $\lambda_{a}$ and $\lambda_{s}$.

For other remodelling and design problems, the inner iteration loop has to be modified according to the optimality conditions.

\section{FINITE ELEMENT DISCRETIZATION}

The numerical treatment of the design problem is based on a discretized model of the design domain and its boundaries. At each iteration step in the computational procedure, the remodelling function $\rho$ is approximated on a line element for the boundaries. The solution to the boundary-value problem (8) is approximated by a finite element method using linear triangular elements. It is noted that the use of a linear finite element draws special attention to the evaluation of the specific strain energy $U$ along the boundaries. This is particularly important because the evaluation of $U$ requires evaluation of the first derivatives of the displacement $\mathbf{u}$. Along the boundaries under the consideration of design modification, the finite element mesh is arranged such that elements are as regular as possible and their centroids are near enough to the boundaries. In this way the specific strain energy evaluated at the centroid of a linear triangular element which has a boundary line element as one of its edges can be regarded as an approximate measure of $U$ at the centre of a boundary line element. In other words, this value of $U$ becomes a representative value for a boundary line element. Then the sets $I_{r}$ and $I_{l}$ are identified in terms of boundary line elements and two constant energy levels $\lambda_{a}$ and $\lambda_{s}$ are computed, from which movements of the centre points of the boundary elements are calculated. Nodal values of the design corrections $d_{r}^{0}$ and $d_{l}^{0}$ are computed through interpolation of movements of the centre points of the boundary line elements. Since this numerical procedure inevitably involves the error in the amounts of material volume for the modification, simple scaling is performed at each iteration step on remodelling $\rho$ to satisfy the global constraints exactly in the sense of finite element discretization.

The whole finite element mesh is regenerated according to the design changes $d_{r}^{0}$ and $d_{l}^{0}$, if they are large enough. If otherwise, only the nodal positions of the boundary nodal points are changed.

\section{EXAMPLE PROBLEMS OF SHAPE REMODELLING}

The solution scheme described is applied to solve several design problems of plane stress in order to demonstrate its capability. Each example problem is solved for a set of values of 
specified amount of material for the modification. The values are given in terms of the ratio of specified amount of material for the modification to the amount of material for the prescribed initial design. The purpose in presenting such a sequence of solution is to demonstrate changes in shape with increasing percentage of modification.

The typical finite element model chosen for a given simply-connected domain is shown in Figure 3. Under the same loading conditions this model can represent two different structures, depending upon the boundary conditions. When the sliding boundary condition is assumed along the boundary identified with the line connecting the points $a-b-c$, the model becomes the one representing one-quarter of the whole structure, so that the lines connecting the points $a-b$ and $b-c$ become lines of symmetry. When the rigidly fixed boundary condition is assumed along the same boundary, the model by itself represents the whole structure. In the former case, shape modification along the boundaries identified with the lines connecting the points $a-e$ and $c-d$ is for the free boundaries. In the latter case, the change of the points ' $a$ ' and ' $c$ ' involves the shape modification along both the free boundaries and the rigidly fixed boundaries, since the points ' $a$ ' and ' $c$ ' belong to the rigidly fixed boundary.

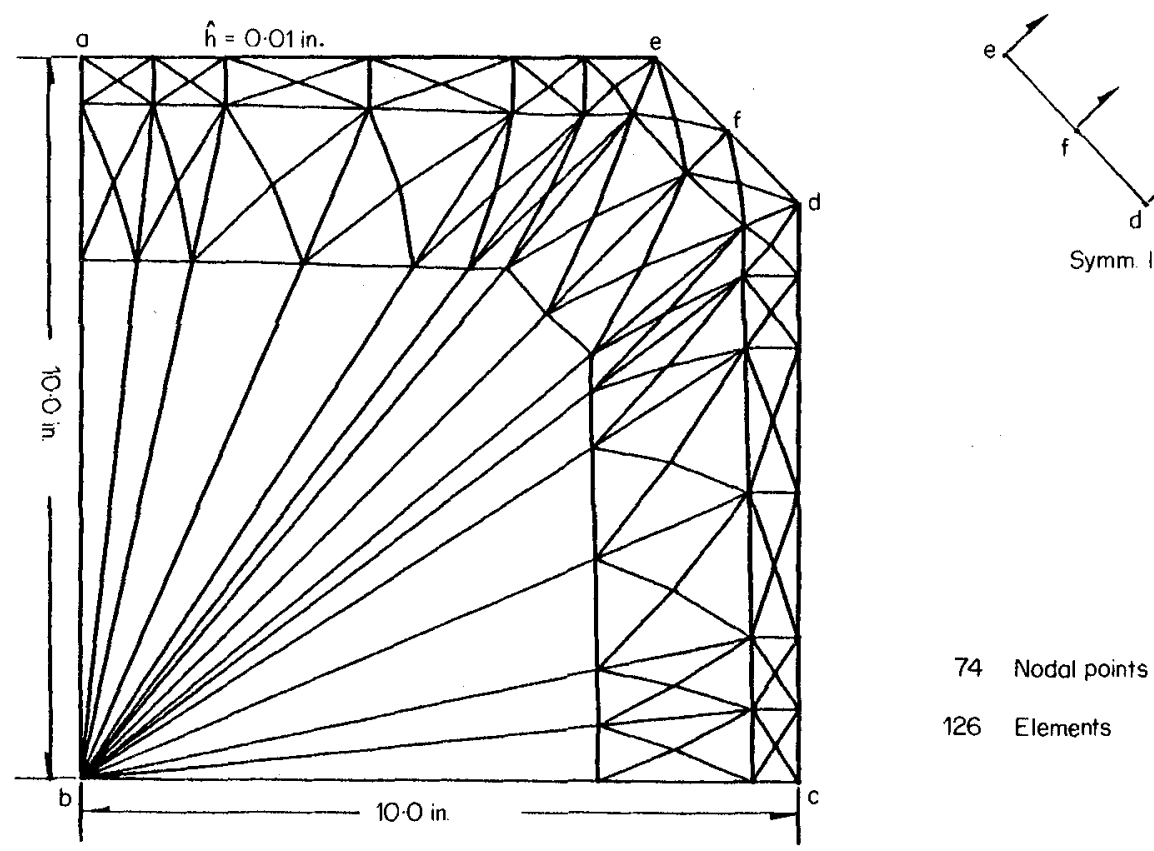

Figure 3. Typical finite element model of a simply-connected domain

The design problem of lightening remodelling is solved for these two different cases under the same loading condition shown in Figure 3, with numerical data of Lame's constants $\lambda=1.2 \times 10^{7} \mathrm{psi}$ and $G=0.8 \times 10^{7} \mathrm{psi}$. Their numerical results are shown in Figures 4 and 5 .

Another simply-connected domain is chosen for the design problem of reinforcement remodelling. Figure 6 shows the typical finite element model representing the one-quarter of a whole structure with the specified loading condition. The lines connecting the points $a-b$ and $\mathrm{b}-\mathrm{c}$, are lines of symmetry. Reinforcement remodelling is considered along the free boundary identified with the line connecting the points $\mathrm{a}-\mathrm{h}-\mathrm{g}-\mathrm{f}$, for the case that a uniform horizontal 


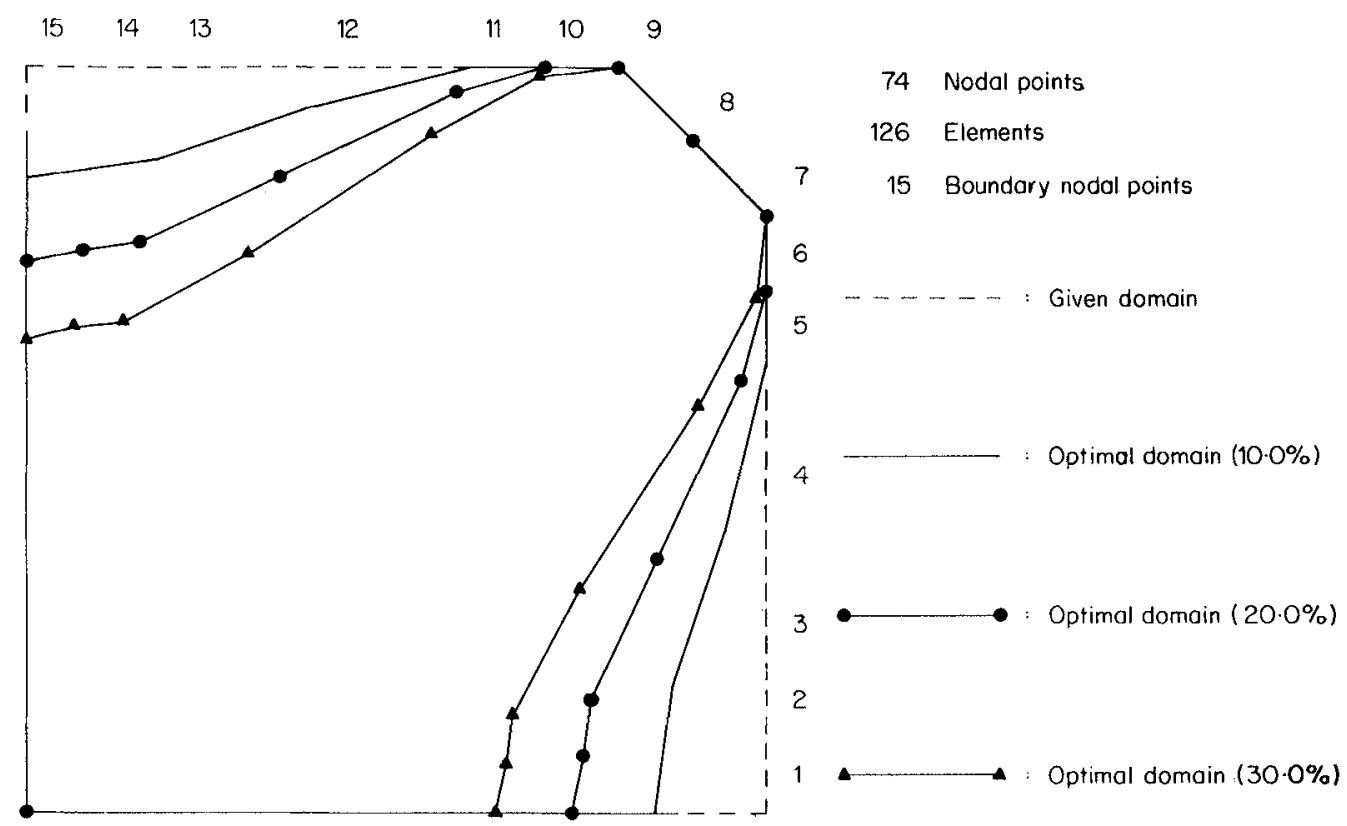

Figure 4. Given and optimal domains: lightening along the free boundary of a simply-connected domain for maximum mean stiffness (sliding condition)

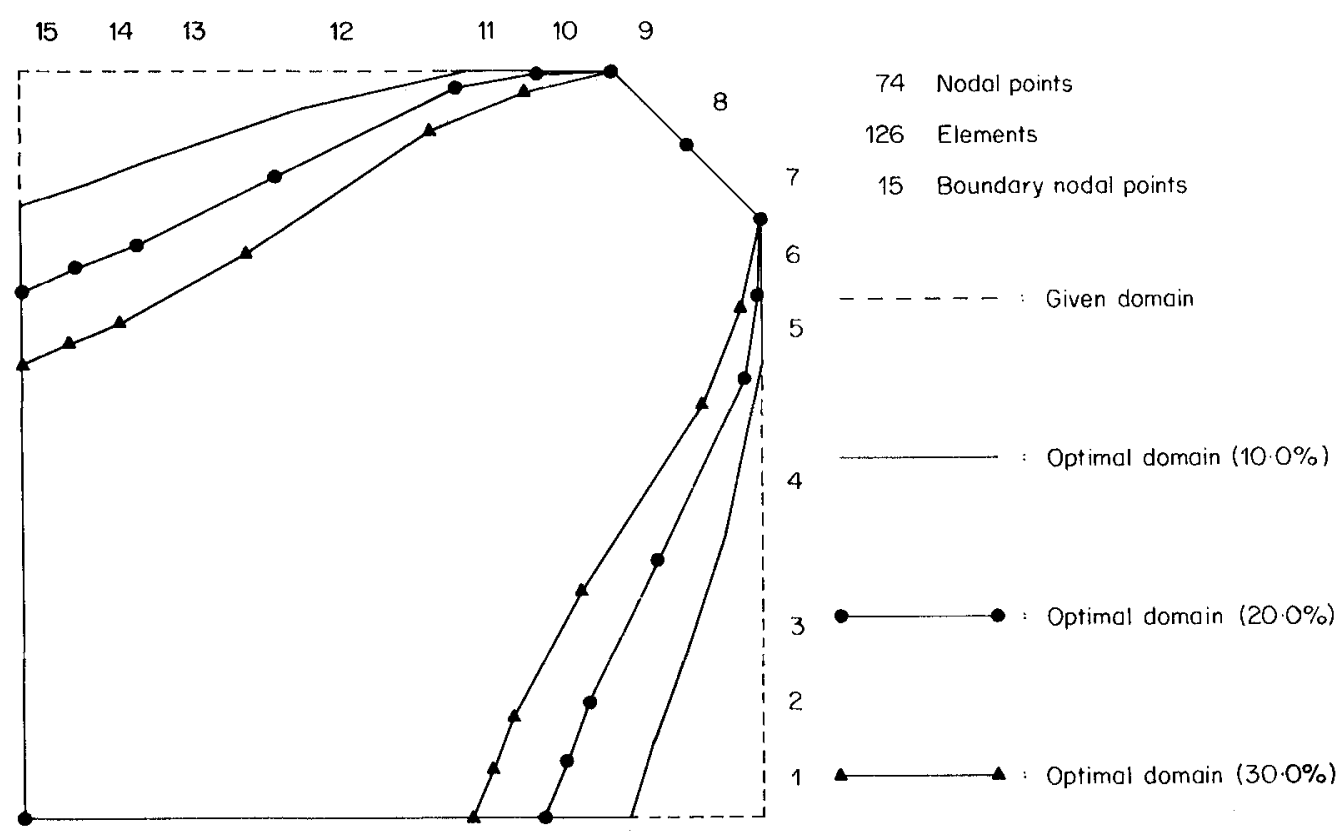

Figure 5. Given and optimal domains: lightening along the boundary of a simply-connected domain for maximum mean stiffness (fixed condition) 


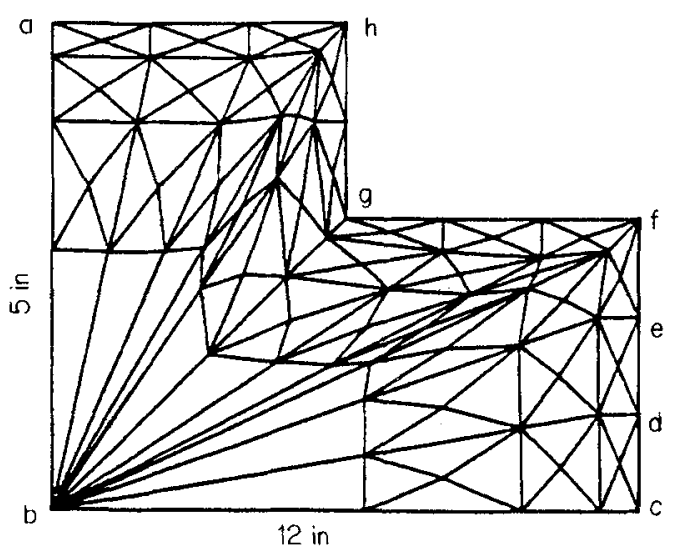

$$
\begin{aligned}
& \text { f } 300 \mathrm{lbf} \\
& \text { e } 600 \mathrm{lbf} \\
& \text { d } 600 \mathrm{lbf} \\
& \text { c } 300 \mathrm{lbf} \\
& \text { thickness }=0.01 \mathrm{in}
\end{aligned}
$$

Figure 6. Typical finite element model of a fillet

traction is applied along the boundary $\mathrm{f}, \mathrm{e}, \mathrm{d}$ and $\mathrm{c}$. The amount of the traction is given in Figure 6 as equivalent nodal forces. The numerical results are shown in Figure 7.

The design problem of lightening remodelling as the generation of a hole is considered for a simply-connected domain, for which Figure 8 shows the typical finite element model with the specified symmetric loading condition. The rigidly fixed boundary condition is assumed along the boundary identified with the line connecting the points $a-b-c$, and the free boundary condition is assumed along the boundaries identified with the lines connecting the points a-e and $\mathrm{c}-\mathrm{d}$. The numerical results are shown in Figure 9.

As the last example problem, the compound remodelling along both the internal and external boundaries is considered for a doubly-connected domain. Here, reinforcement, lightening or a combination of both can occur either along the free external boundary or the free internal boundary. The typical finite element model for one-quarter of a whole structure is shown in

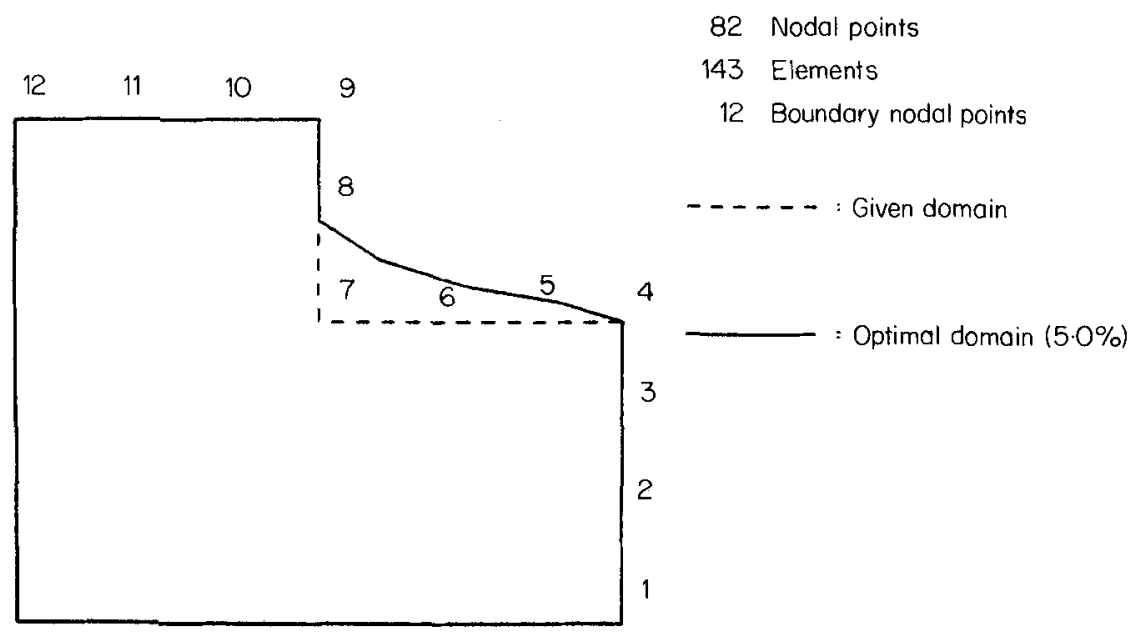

Figure 7. Given and optimal domains: reinforcement along the free boundary of a fillet 


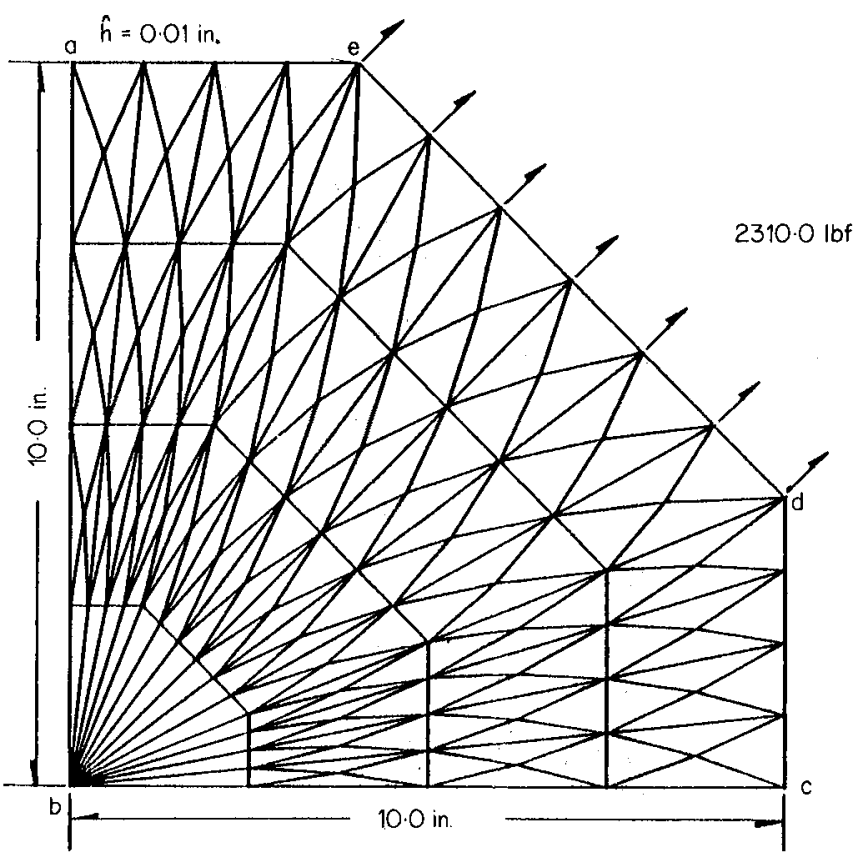

103 Nodal points

182 Elements

Figure 8. Typical finite element model of a simply-connected domain: starting model for generation of a hole

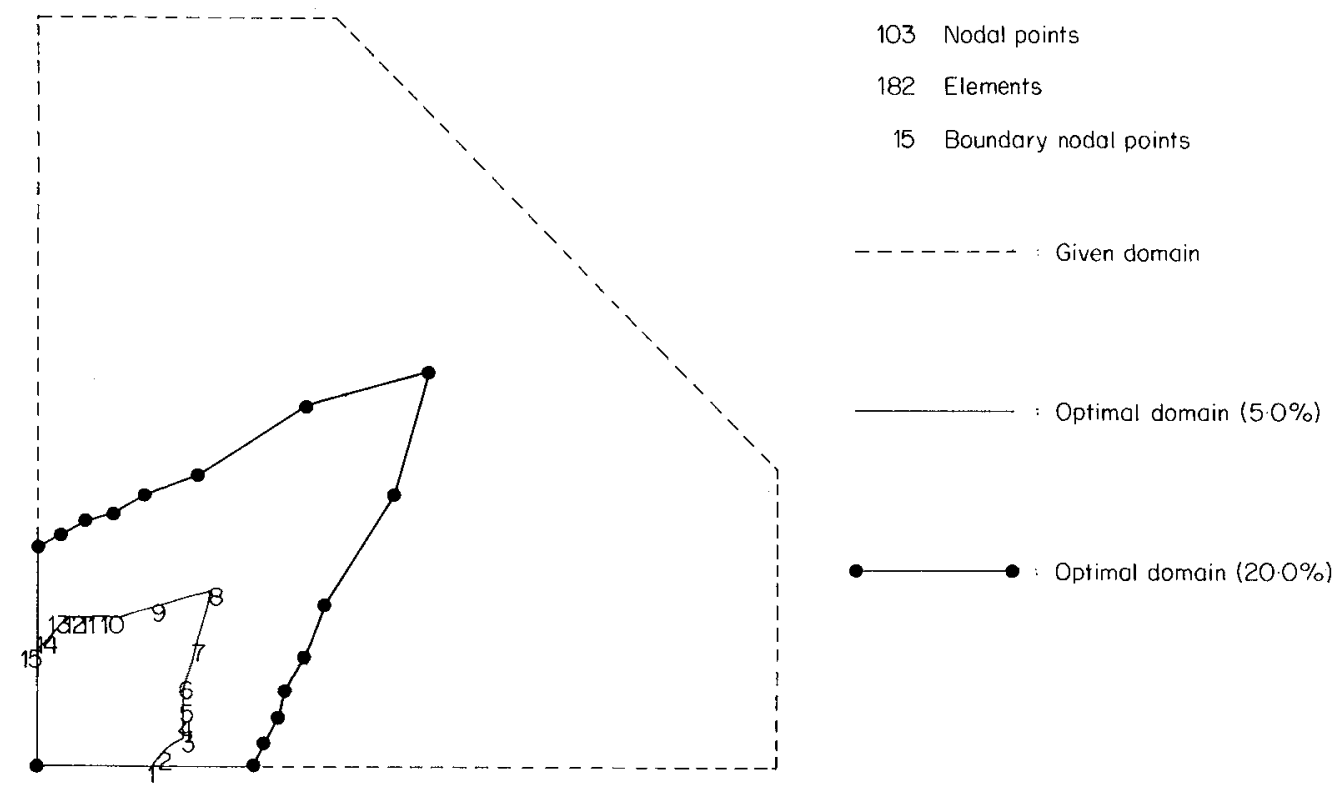

Figure 9. Given and optimal domains: hole generation inside a simply-connected domain for maximum mean stiffness 


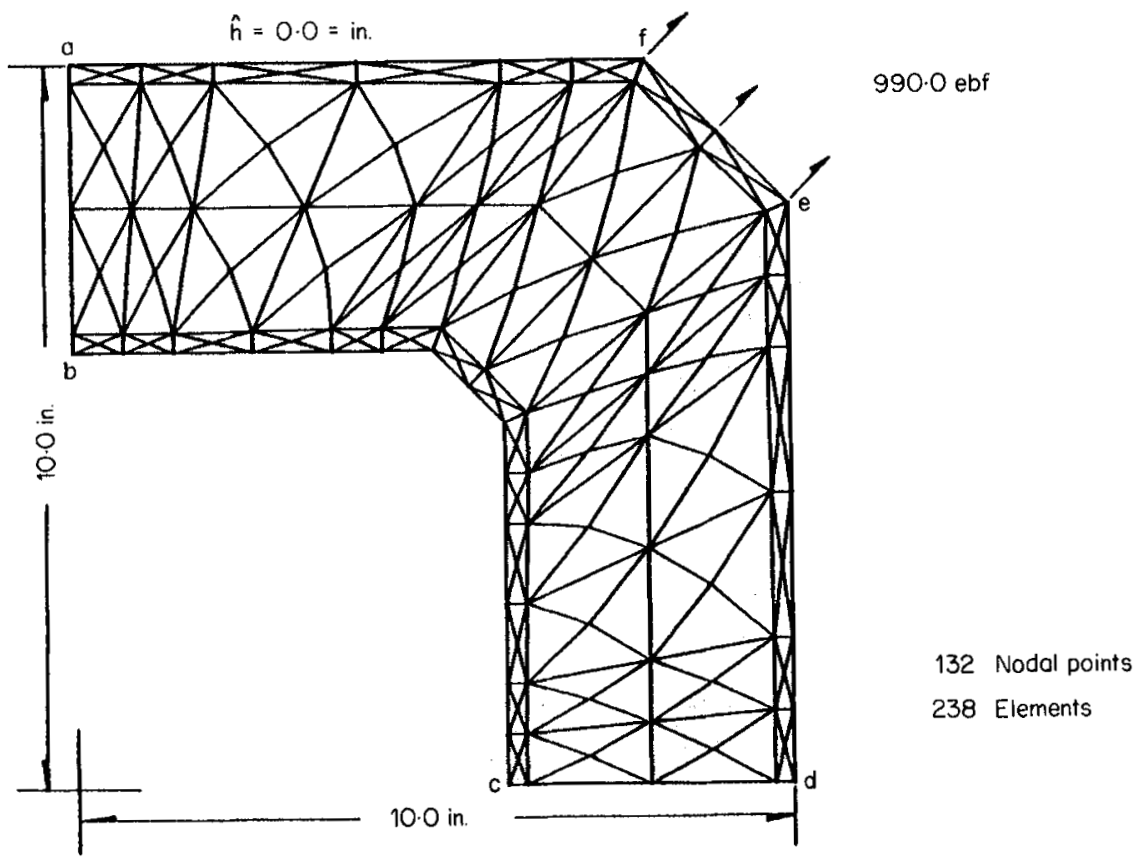

Figure 10. Typical finite element model of a doubly-connected domain
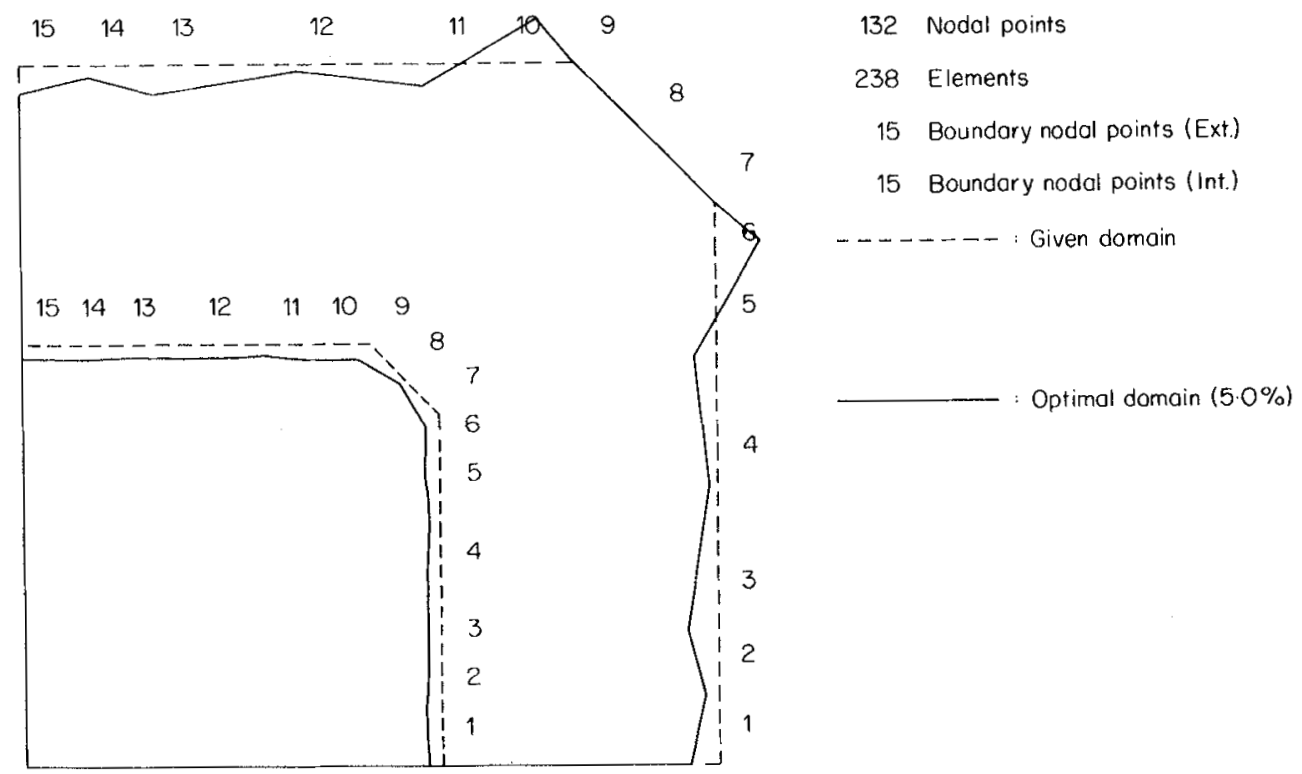

Figure 11. Given and optimal domains: compound remodelling along the free boundaries of a doubly-connected domain for maximum mean stiffness 
Figure 10 with the specified loading condition. The lines connecting the points $a-b$ and $c-d$ are lines of symmetry. The numerical results are displayed in Figure 11.

Numerical results obtained so far show the capability of the proposed solution scheme to predict optimal shapes, even with relatively crude finite element models and with approximations related to the computation of specific strain energy distributions along the boundaries.

It should be noted that, for almost all of the cases, mean compliance has reached its minimum just after a few steps of iteration-between 6 and 10, depending upon the problem. However, it takes more iteration steps to achieve constancy of specific strain energy along the parts of boundaries where the shape modification is made. This means that a small change in the shape does not induce a substantial change in mean compliance.

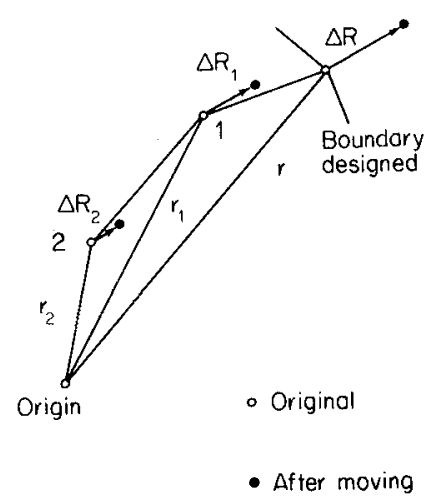

Figure 12. Movement of internal nodes

It is also worth while to describe a method to move internal nodes of the finite element model according to the design change of each iteration. In the above examples, a projection method shown in Figure 12 is applied. If the amount of the movement of a node on the boundary is $\Delta R$ in the direction $\mathbf{N}$, then the nodes chained to the boundary node from the origin is shifted by the rule

$$
\Delta R_{i}=\Delta R r_{i} / r
$$

Here $r_{i}$ is the distance of the $i$ th node from the origin, $r$ the distance of the boundary node from the origin, and $\Delta R_{i}$ is the amount of the movement of the $i$ th node. The direction of the movement of each node follows the one of the boundary node. This projected method, in general, provides similar accuracy on the finite element approximation at each iteration to the one for the initial design step.

Application of the same solution method to torsion problems can be found in $\mathrm{Na}$ et al. ${ }^{15}$

\section{ACKNOWLEDGEMENTS}

During the present work, the authors were supported by the NSF under Grant CEE 8118158. The authors also express their appreciation to the reviewer whose suggestions improve the quality of the paper. 


\section{REFERENCES}

1. W. Prager, 'Conditions for structural optimality', Comp. Struct. 2, 833-840 (1972).

2. O. C. Zienkiewicz and J. S. Campbell, 'Optimum structural design', in Shape Optimization and Sequential Linear Programming (Eds. R. H. Gallagher and O. C. Zienkiewicz), Wiley, London, 1973.

3. A. Francavilla, C. V. Ramakrishnan and O. C. Zienkiewicz, 'Optimization of shape to minimize stress concentration', J. Strain Anal. 10(2), 63-70 (1975).

4. K. Dems and Z. Mroz, 'Multiparameter structural shape optimization by the finite element method', Int. j. numer. methods eng, 13, 247-263 (1978).

5. E. J. Haug, K. K. Choi, J. W. Hou and Y. M. Yoo, 'A variational method for shape optimal design of elastic structures', Int. Symp. on Optimum Structural Design, 19-22 October, Tucson, Arizona (1981).

6. C. V. Ramakrishnan and A. Francavilla, 'Structural shape optimization using penalty functions', J. Struct. Mech. 3(4), 403-422 (1974-75).

7. E. S. Kristensen and N. F. Madsen, 'On the optimum shape of fillets in plates subjected to multiple inplane loading cases', Int. j. numer. methods eng, 10, 1006-1019 (1976).

8. Y. W. Chun and E. J. Haug, 'Two-dimensional shape optimal design', Int. j. numer. methods eng, 13, 311-336 (1978).

9. J. P. Queau and P. H. Trompette, 'Two-dimensional shape optimal design by the finite element method', Int. j. numer. methods eng, 15, 1603-1612 (1980).

10. M. H. Imam, 'Three-dimensional shape optimization', Int. j. numer. methods eng, 18, 661-673 (1982).

11. E. J. Haug, 'A review of distributed parameter structural optimization literature', in Optimization of Distributed Parameter Structures (Eds. E. J. Haug and J. Cea), Sitjhoff and Noordhoff, The Netherlands, 1981.

12. N. Olhoff and J. E. Taylor, 'On optimal structural remodelling', J. Optim. Theory Appl. 27(4), 571-582 (1979).

13. M. S. Na, 'Optimal shape remodelling of elastic bodies by the finite element method', Ph.D. thesis, Univ. of Michigan, Ann Arbor (1982).

14. H. Uzawa, 'Iterative methods for concave programming', in Studies in Linear and Non-linear Programming (Eds. K. J. Arrow, L. Hurwitz and H. Uzawa), Stanford University Press, 1958, pp. 154-165.

15. M. S. Na, N. Kikuchi and J. E. Taylor, 'Optimal modification of shape for two-dimensional elastic bodies', $J$. Struct. Mech., 11, 111-135 (1983). 